\title{
Aboriginal and Torres Strait Islander communities forgotten in new Australian National Action Plan for Human Influenza Pandemic: "Ask us, listen to us, share with us"
}

Adrian Miller and David N Durrheim on behalf of the Aboriginal and Torres Strait Islander Community Influenza Study Group

\author{
The epidemiology of influenza pandemics demands that Aboriginal and \\ Torres Strait Islander people occupy centrestage in future planning
}

$\mathrm{T}$ he first wave of pandemic (H1N1) 2009 influenza (pH1N1) broke more heavily on Australia's Aboriginal and Torres Strait Islander populations than on non-Indigenous Australians. The burden of disease in Aboriginal and Torres Strait Islander people was highlighted by the first Australian death associated with pH1N1 infection: a young Aboriginal man from a remote area of Western Australia who died on 19 June 2009 in an Adelaide hospital. ${ }^{1}$ The differences between the populations are stark, with Aboriginal and Torres Strait Islander people indisputably over-represented in severe $\mathrm{pH} 1 \mathrm{Nl}$ disease. In the Top End of the Northern Territory, pH1N1 rates of notification, hospital admission and intensive care unit (ICU) admission were higher for Aboriginal and Torres Strait Islander people than for the nonIndigenous population (3.5 times, 12 times and 5 times, respectively). ${ }^{2}$ Similar profound differences have been recorded for Aboriginal communities in New South Wales: Aboriginal people hospitalised with $\mathrm{pH} 1 \mathrm{~N} 1$ were younger than their non-Aboriginal counterparts (median age of 24.5 years compared with 31.7 years), and the age-standardised rate ratios for Aboriginal to non-Aboriginal admissions to hospital, admissions to ICU and death during the 2009 pandemic wave were 3.2, 4.0 and 4.5, respectively. ${ }^{3}$

Overall, from May to October 2009 in Australia, Aboriginal and Torres Strait Islander Australians, who comprise 2.5\% of the population, accounted for $16.0 \%$ of hospitalisations with pH1N1 and $9.7 \%$ of pH1N1 admissions to an ICU. ${ }^{4}$ A fivefold increase in risk of death due to pHlNl was also reported. ${ }^{5}$ This experience demands a greater focus on the needs of Aboriginal and Torres
Strait Islander communities and their prioritisation in future pandemic planning. We should not have been surprised, as history tragically demonstrates disproportionate morbidity and mortality for Aboriginal and Torres Strait Islander people in previous pandemics. ${ }^{6}$

It is thus exceedingly disappointing to discover no mention of Aboriginal and Torres Strait Islander Australians in the revised National Action Plan for Human Influenza Pandemic (NAP). ${ }^{7}$ The 2010 NAP fails to identify Aboriginal and Torres Strait Islander people as a high-risk group during the H1N1 2009 pandemic, although it acknowledges other risk groups that have been recognised internationally and in Australia:

severe cases occurred in people with underlying chronic conditions such as respiratory diseases, cardiovascular disease, diabetes, autoimmune disorders and obesity. Pregnant women were also at an increased risk of serious disease. ${ }^{7}$

It is inexplicable that while Aboriginal and Torres Strait Islander people were identified as a priority group for the rollout of the pH1N1 influenza vaccination - a commendable and necessary preventive strategy - they are overlooked in the NAP. ${ }^{8}$ Although the Australian Health Management Plan for Pandemic Influenza ${ }^{9}$ states an equity commitment, and a subsequent appendix ${ }^{10}$ produced during the "Protect" phase of the 2009 pandemic endorsed the need for partnership between all health care providers in case and contact management among the Aboriginal and Torres Strait Islander population, respectful partnership between governments 
and Aboriginal and Torres Strait Islander communities to identify culturally appropriate and effective prevention and mitigation strategies enjoys no mention.

Given that the NAP is the peak plan for guiding preparations for future pandemics, there is a fundamental need for governments to acknowledge and respond effectively to the specific requirements of Aboriginal and Torres Strait Islander people. Prevention and preparedness must include government support of planning in respectful partnership with Aboriginal and Torres Strait Islander communities, health organisations and representative bodies. Mandating this support and partnership at all levels of government will allow a greater understanding of infection risk and identification of cultural, social, economic and health service factors that may contribute to poor health outcomes, and ensure culturally safe and effective prevention and mitigation strategies.

A national project, funded by the National Health and Medical Research Council, working with Aboriginal and Torres Strait Islander communities and health services in NSW, Queensland and Western Australia is learning about feasible and culturally appropriate containment strategies. ${ }^{11}$ A strong theme emerging from this work is the message to government: "Ask us, listen to us, share with us". The ability of Aboriginal and Torres Strait Islander communities to develop novel practical mitigation measures has been a particular feature of this respectful engagement that has already informed government strategies in NSW. ${ }^{3}$

The epidemiology of the current and previous influenza pandemics demands that Aboriginal and Torres Strait Islander people occupy centrestage in future planning. Solutions to limit the burden on Aboriginal and Torres Strait Islander populations exist, but respectful partnership is necessary to unearth them. The partnership must not be a token one, but one developed through engagement with communities, and with the flexibility to be localised to meet the specific needs of diverse urban, rural and remote Aboriginal and Torres Strait Islander communities in all states and territories. Health information delivered with a local flavour is a key message from the project. "Ask us, listen to us, share with us" is a strong message that governments must heed if the impact of pandemic influenza on Aboriginal and Torres Strait Islander communities is to be limited.

\section{Acknowledgements}

The Aboriginal and Torres Strait Islander Community Influenza Study Group is supported by a National Health and Medical Research Council H1N1 competitve grant no. 601034. Members of the Aboriginal and Torres Strait Islander Community Influenza Study Group are: New South Wales: David Durrheim, University of Newcastle; Glenn Pearce, Kylie Taylor, Hunter New England Population Health; Peter Massey, University of New England. Queensland: Magdalena Blackley, Research Assistant, Palm Island; Jennifer Broome, Travis Odo, Colleen Purcell, Mamu Health Service; Alan Clough, Jenni Judd, Jenny Kelly, Adrian Miller, Richard Speare, James Cook University. Western Australia: Sherry Saggers, Curtin University of Technology.

\section{Competing interests}

None identified.

\section{Author details}

Adrian Miller, BA, MPH, Senior Lecturer ${ }^{1}$

David N Durrheim, MB ChB, FAFPHM, DrPH, Professor of Public Health and Medical Practice ${ }^{2}$

1 Anton Breinl Centre for Public Health and Tropical Medicine, James

Cook University, Townsville, QLD.

2 University of Newcastle, Newcastle, NSW.

Correspondence: Adrian.Miller@jcu.edu.au

\section{References}

1 Department of Health and Ageing. H1N1 09 update bulletin 5 am, 20 June 2009. Canberra: Commonwealth of Australia, 2009. http:// www.healthemergency.gov.au/internet/healthemergency/publishing.nsf/ Content/87EACFF5730CFA33CA25761C00042589/\$File/H1N1 \%20Influenza\%2009\%205am\%2020\%20June.pdf (accessed Jun 2010).

2 Flint SM, Davis JS, Su J, et al. Disproportionate impact of pandemic (H1N1) 2009 influenza on Indigenous people in the Top End of Australia's Northern Territory. Med J Aust 2010; 192: 617-622.

3 Rudge S, Massey PD. Responding to pandemic (H1N1) 2009 influenza in Aboriginal communities in NSW through collaboration between NSW Health and the Aboriginal community-controlled health sector. N S W Public Health Bull 2010; 21: 26-29.

4 Bishop JF, Murnane MP, Owen R. Australia's winter with the 2009 pandemic influenza A (H1N1) virus. N Engl J Med 2009; 361: 2591-2594.

5 Kelly H, Mercer GN, Cheng AC. Quantifying the risk of pandemic influenza in pregnancy and Indigenous people in Australia in 2009. Euro Surveill 2009; 14: pii:19441.

6 Cleland Burton J. Disease among the Australian Aborigines. J Trop Med Hyg 1928; 6: 65 .

7 Department of the Prime Minister and Cabinet. National Action Plan for Human Influenza Pandemic. April 2010. Canberra: Commonwealth of Australia, 2010. http://www.dpmc.gov.au/publications/pandemic/docs/ NAP.pdf (accessed Jun 2010).

8 Department of Health and Ageing. Pandemic (H1N1) 2009 vaccination program. Information for heath professionals. Updated 3 December 2009. Canberra: Commonwealth of Australia, 2009. http:// www.health.gov.au/internet/healthemergency/publishing.nsf/Content/ 2723D54E78BF5739CA2576400027E1E9/\$File/information-sheet031209.pdf (accessed Jun 2010).

9 Department of Health and Ageing. Australian Health Management Plan for Pandemic Influenza 2008. Canberra: Commonwealth of Australia, 2008. http://www.flupandemic.gov.au/internet/panflu/publishing.nsf/ Content/8435EDE93CB6FCB8CA2573D700128ACA/\$File/Pandemic\%20FINAL\%20webready.pdf (accessed Aug 2010).

10 Department of Health and Ageing. Appendix to the PROTECT Annex of the Australian Health Management Plan for Pandemic Influenza. Guidance for primary health care workers providing care to Aboriginal and Torres Strait Islander people. 8 July 2009. Canberra: Commonwealth of Australia, 2009. http://www.health.gov.au/internet/healthemergency/ publishing.nsf/Content/resources/\$file/Indigenous\%20Appendix\% 20V1\%209\%20July\%202009.pdf (accessed Jul 2010).

11 Massey PD, Pearce G, Taylor KA, et al. Reducing the risk of pandemic influenza in Aboriginal communities. Rural Remote Health 2009; 9: 1290. ـ 